\title{
MINIMAL PAIRS OF COMPACT CONVEX SETS
}

\author{
DIETHARD PALLASCHKE \\ Institut für Statistik und Mathematische Wirtschaftstheorie, Universität Karlsruhe \\ Kaiserstr. 12, D-76128 Karlsruhe, Germany \\ RYSZARD URBAŃSKI \\ Wydziat Matematyki i Informatyki, Uniwersytet im. Adama Mickiewicza \\ Umultowska 87, PL-61-614 Poznań, Poland \\ E-mail: rich@amu.edu.pl
}

\begin{abstract}
Pairs of compact convex sets naturally arise in quasidifferential calculus as sub- and superdifferentials of a quasidifferentiable function (see [1]). Since the sub- and superdifferentials are not uniquely determined, minimal representations are of special importance. In this paper we give a survey on some recent results on minimal pairs of closed bounded convex sets in a topological vector space (see [11]). Particular attention is paid to the problem of characterizing minimal representatives of a pair of nonempty compact convex subsets of a locally convex topological vector space in the sense of the Rådström-Hörmander theory.
\end{abstract}

1. Notations and preliminaries. For a topological vector space $X=(X, \tau)$ let us denote by $\mathcal{A}(X)$ the set of all nonempty subsets of $X$, by $\mathcal{B}^{*}(X)$ the set of all nonempty bounded subsets of $X$, by $\mathcal{C}(X)$ the set of all nonempty closed convex subsets of $X$, by $\mathcal{B}(X)=\mathcal{B}^{*}(X) \cap \mathcal{C}(X)$ the set of all bounded closed convex sets of $X$ and by $\mathcal{K}(X)$ the set of all nonempty compact convex subsets of $X$. For $A, B \in \mathcal{A}(X)$ the algebraic sum is defined by $A+B=\{x=a+b \mid a \in A$ and $b \in B\}$ and for $\lambda \in \mathbb{R}$ and $A \in \mathcal{A}(X)$ the multiplication is defined by $\lambda A=\{x=\lambda a \mid a \in A\}$. The Minkowski sum for $A, B \in \mathcal{A}(X)$ is defined by

$$
A \dot{+} B=\operatorname{cl}(\{x=a+b \mid a \in A \text { and } b \in B\}),
$$

where $\operatorname{cl}(A)=\bar{A}$ denotes the closure of $A \subset X$ with respect to $\tau$. By relint $(A)$ we denote the relative interior of $A \subset X$ with respect to $\tau$.

2000 Mathematics Subject Classification: 26A27, 90C30.

Key words and phrases: quasidifferentiable function, pairs of compact convex sets.

The paper is in final form and no version of it will be published elsewhere. 
For $A, B \in \mathcal{A}(X)$ we define: $A \stackrel{\circ}{\vee} B=\operatorname{conv}(A \cup B), A \vee B=\overline{A \stackrel{\circ}{\vee} B}=\operatorname{cl} \operatorname{conv}(A \cup B)$ and by $A \underline{\vee} B=\bigcup_{\alpha, \beta \geq 0, \alpha+\beta=1}(\alpha A+\beta B)$ the skeleton of $A$ and $B$. It is easy to observe that $A \underline{\vee} B \subset A \stackrel{\circ}{\vee} B \subset A \vee B$. In the case when $A$ and $B$ are convex sets then $A \underline{\vee} B=$ $A \stackrel{\circ}{\vee} B$. For two elements $a, b \in X$ the interval with end points $a$ and $b$ will be denoted by $[a, b]=\{a\} \vee\{b\}$.

For compact convex sets, the Minkowski sum coincides with the algebraic sum, i,e., for $A, B \in \mathcal{K}(X)$ we have $A \dot{+} B=A+B$ and also $A \stackrel{\circ}{\vee} B=A \vee B$. We will use the abbreviation $A \dot{+} B \vee C$ for $A \dot{+}(B \vee C)$ and $C+d$ instead of $C+\{d\}$ for all bounded closed convex sets $A, B, C \in \mathcal{A}(X)$ and a point $d \in X$.

A convex subset $B$ of a convex set $A \subseteq X$ is called an extreme subset if for every $x, y \in A$ and some $t \in(0,1)$ the condition $t x+(1-t) y \in B$ implies that $x, y \in B$. An extreme subset which consists of a single point only is called an extreme point and $\mathcal{E}(A)$ denotes the set of extreme points of $A$.

A convex set which is the convex hull of finitely many points is called a polytope. The set of all polytopes of a vector space $X$ is denoted by $\mathcal{P}(X)$. An extreme subset of a polytope is called a face and a one-dimensional extreme set of a polytope is called an edge.

If $(X, \tau)$ is a topological vector space and $X^{*}$ its dual space, then for $A \in \mathcal{K}(X)$ and $f \in X^{*}$ we denote by

$$
H_{f}(A)=\left\{z \in A \mid f(z)=\max _{y \in A} f(y)\right\}
$$

the (maximal) face of $A$ with respect to $f$.

Finally, we will call a set $A \in \mathcal{B}(X)$ a summand of $B \in \mathcal{B}(X)$ if there exists a set $C \in \mathcal{B}(X)$ such that $A \dot{+} C=B$.

The following statements hold for convex sets:

Addition of maximal faces:

Proposition 1.1. Let $X$ be a topological vector space, $f \in X^{*}$ and $A, B \in \mathcal{K}(X)$. Then

$$
H_{f}(A+B)=H_{f}(A)+H_{f}(B) .
$$

Proof. Assume that $x=a+b \in H_{f}(A+B)$ with $a \in A$ and $b \in B$. Then $a \in H_{f}(A)$ and $b \in H_{f}(B)$. Indeed, assume for instance that $a \notin H_{f}(A)$. Since $A \in \mathcal{K}(X)$ is compact, there exists an element $a^{\prime} \in A$ with $f(a)<f\left(a^{\prime}\right)$. From this it follows that

$$
f(x)=f(a)+f(b)<f\left(a^{\prime}\right)+f(b)=f\left(a^{\prime}+b\right) \leq \sup _{\substack{u \in A \\ v \in B}} f(u+v)=f(x)
$$

because $x \in H_{f}(A+B)$. This implies the inclusion

$$
H_{f}(A+B) \subseteq H_{f}(A)+H_{f}(B) .
$$

The reverse inclusion can be proved in the same way. Assume that $a \in H_{f}(A)$ and $b \in H_{f}(B)$. Then $x=a+b \in H_{f}(A+B)$. Let us assume that this is not true. Then there exists an element $x^{\prime}=a^{\prime}+b^{\prime} \in A+B$ with $f(x)<f\left(x^{\prime}\right)$. But this implies

$$
f(a)+f(b)=f(x)<f\left(x^{\prime}\right)=f\left(a^{\prime}\right)+f\left(b^{\prime}\right)
$$

and hence $f(a)<f\left(a^{\prime}\right)$ or $f(b)<f\left(b^{\prime}\right)$, which completes the proof. 
Additivity of the convex hull:

Proposition 1.2. Let $X$ be a vector space and $A, B \subset X$. Then

$$
\operatorname{conv} A+\operatorname{conv} B=\operatorname{conv}(A+B) \text {. }
$$

Proof. First observe that

$$
\begin{aligned}
\operatorname{conv} A+B & =\bigcup_{b \in B}(\operatorname{conv} A+b)=\bigcup_{b \in B}[\operatorname{conv}(A+b-b)+b] \\
& \subseteq \bigcup_{b \in B}(\operatorname{conv}[\operatorname{conv}(A+B)-b]+b)=\operatorname{conv}(A+B) .
\end{aligned}
$$

Since $A+B \subset \operatorname{conv} A+\operatorname{conv} B$ we have $\operatorname{conv}(A+B) \subset \operatorname{conv} A+\operatorname{conv} B$. Now it follows from the above observation that

$$
\begin{aligned}
\operatorname{conv}(A+B) & \subseteq \operatorname{conv} A+\operatorname{conv} B \subseteq \operatorname{conv}(A+\operatorname{conv} B) \\
& \subseteq \operatorname{conv}[\operatorname{conv}(A+B)]=\operatorname{conv}(A+B) .
\end{aligned}
$$

2. The ordered semigroup of convex sets. From the algebraic point of view the set $\mathcal{B}(X)$ of all nonempty closed bounded convex subsets of a real topological vector space $(X, \tau)$, endowed with the Minkowski addition, is a commutative semigroup which contains $\mathcal{K}(X)$, i.e. the set all nonempty compact convex subsets, as a sub-semigroup. Both semigroups $\mathcal{B}(X)$ and $\mathcal{K}(X)$ are ordered by inclusion, i.e. for $A, B \in \mathcal{B}(X), A \leq B$ if and only if $A \subseteq B$.

Now we state two fundamental properties of the semigroups $\mathcal{B}(X)$ and $\mathcal{K}(X)$, the order cancellation law [13], [16] and Pinsker's formula [12].

The order cancellation law:

Theorem 2.1. Let $X$ be a topological vector space. Then for any $A \in \mathcal{A}(X), B \in \mathcal{B}^{*}(X)$ and $C \in \mathcal{C}(X)$ the inclusion

$$
A+B \subseteq C+B \quad \text { implies } A \subseteq C .
$$

Proof. Let $\mathcal{U}$ be a base of neighborhoods of zero in the topological vector space $X$. Given any neighborhood $U \in \mathcal{U}$ we define a sequence $\left(V_{n}\right)_{n \in \mathbb{N}}$ such that

$$
V_{0}+V_{0} \subseteq U \text { and } V_{n+1}+V_{n+1} \subseteq V_{n}
$$

From $A+B \subseteq C \dot{+} B$ it follows that for every $V \in \mathcal{U}$ we have

$$
A+B \subseteq C+B+V
$$

and hence for every $n \in \mathbb{N}$ we have

$$
A+B \subseteq C+B+V_{n} .
$$

Now let $a \in A$ and $b_{1} \in B$. Then

$$
\begin{aligned}
& a+b_{1}=c_{1}+b_{2}+v_{1} \quad \text { for some } c_{1} \in C, b_{2} \in B, v_{1} \in V_{1} \text {, } \\
& a+b_{2}=c_{2}+b_{3}+v_{2} \quad \text { for some } c_{2} \in C, b_{3} \in B, v_{2} \in V_{2} \text {, }
\end{aligned}
$$

and in general, for every $n \in \mathbb{N}$,

$$
a+b_{n}=c_{n}+b_{n+1}+v_{n} \quad \text { for some } c_{n} \in C, b_{n+1} \in B, v_{n} \in V_{n} .
$$


Hence

$$
a=\frac{1}{n}\left(c_{1}+\ldots+c_{n}\right)+\frac{1}{n}\left(b_{n+1}-b_{1}\right)+\frac{1}{n}\left(v_{1}+\ldots+v_{n}\right), \quad n \in \mathbb{N}
$$

and thus by the convexity of $C$ and the boundedness of $B$ we get for sufficiently large $n \in \mathbb{N}$ that

$$
a \in C+V_{0}+V_{1}+\ldots+V_{n} \subseteq C+U .
$$

Thus $A \subseteq C+U$ for every $U \in \mathcal{U}$, and therefore, $A \subset C$.

The implication $A+B \subseteq C+B \Rightarrow A \subseteq C$ is called the order cancellation law and the weaker implication $A+B=C+B \Rightarrow A=C$ is called the cancellation law.

The Pinsker formula:

Next we prove an identity for bounded closed convex sets which was first observed by A. G. Pinsker [12] for locally convex vector spaces and will be called the Pinsker formula. For its proof we need the following three lemmas:

Lemma 2.2. Let $X$ be a vector space and $A, B, C \subset X$ subsets. Then

$$
A \cup B+C=(A+C) \cup(B+C) .
$$

Proof. For $x \in A \cup B+C$, there exist $c \in C$ and $d \in A \cup B$ such that $x=c+d$. Hence $x \in(A+C) \cup(B+C)$, i.e. $A \cup B+C \subseteq(A+C) \cup(B+C)$.

Conversely, for $x \in(A+C) \cup(B+C)$ there exist elements $c \in C$ and $d \in A$ or $d \in B$ such that $x=c+d$. Hence $x \in A \cup B+C$, i.e. $(A+C) \cup(B+C) \subseteq A \cup B+C$.

Lemma 2.3. Let $X$ be a vector space, $A, B, C \in \mathcal{A}(X)$ and $C$ be a convex set. Then

$$
\operatorname{conv}(A \cup B)+C=\operatorname{conv}[(A+C) \cup(B+C)] .
$$

Proof. From Lemma 2.2 and Proposition 1.2 it follows that

$$
\operatorname{conv}[(A+C) \cup(B+C)]=\operatorname{conv}[(A \cup B)+C]=\operatorname{conv}(A \cup B)+C .
$$

Lemma 2.4. Let $X$ be a topological vector space, and $A, B, C \in \mathcal{A}(X)$ and $C$ be a convex set. Then

$$
((A \dot{+} C) \vee(B \dot{+} C))=C \dot{+}(A \stackrel{\circ}{\vee} B) \text {. }
$$

Proof. By Lemma 2.3 we have:

$$
\begin{aligned}
C \dot{+} \operatorname{conv}(A \cup B) & =\operatorname{cl}(\operatorname{cl}(\operatorname{conv}(A \cup B)+\operatorname{cl}(C))=\operatorname{cl}(\operatorname{conv}(A \cup B)+C) \\
& =\operatorname{cl} \operatorname{conv}((A+C) \cup(B+C)) \\
& =\operatorname{cl} \operatorname{conv}(\operatorname{cl}((A+C) \cup(B+C))) \\
& =\operatorname{cl} \operatorname{conv}(\operatorname{cl}(A+C) \cup \operatorname{cl}(B+C)),
\end{aligned}
$$

since for every $D \subseteq X$ we have $\operatorname{cl} \operatorname{conv}(D)=\operatorname{cl} \operatorname{conv}(\operatorname{cl} D)$.

This implies the Pinsker formula:

Proposition 2.5. Let $(X, \tau)$ be a topological vector space, $A, B, C \in \mathcal{A}(X)$ and $C$ be a convex set. Then

$$
(A \dot{+} C) \vee(B \dot{+} C)=C \dot{+}(A \vee B) .
$$

Altogether we can state: 
THEOREM 2.6. Let $(X, \tau)$ be a topological vector space. Then $(\mathcal{B}(X), \dot{+}, \leq)$ is a commutative ordered semigroup with satisfies the order cancellation law and contains $\mathcal{K}(X)$ as a sub-semigroup. Moreover the distributivity law holds for the maximum operation and the Minkowski addition.

3. Order cancellation law and the separation law. The order cancellation law represents an algebraic property of the semigroup $\mathcal{B}(X)$. There exists an equivalent geometric property for closed bounded convex sets which we will call the separation law. Therefore let $A, B$, and $S$ be nonempty subsets of a vector space $X$. We say that the set $S$ separates the sets $A$ and $B$ if $[a, b] \cap S \neq \emptyset$ for every $a \in A$ and $b \in B$. The separation of two sets by another set is a natural generalization of the separation of two disjoint sets by a hyperplane. Note that in this general case the sets $A$ and $B$ can have a nonempty intersection. If a set $S$ separates the sets $A$ and $B$, then $A \cap B \subset S$. The set $S$ is called minimal separating if $S$ separates $A$ and $B$ and if for every $S^{\prime} \subseteq S$ which also separates $A$ and $B$ we have $S^{\prime}=S$.

This definition of separation of two sets by another set is illustrated in Fig. 1.

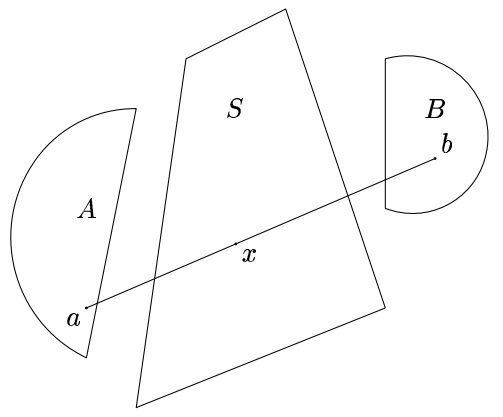

Fig. 1

THEOREM 3.1. Let $A, B$ be nonempty subsets of a topological vector space $X$ and assume that $A \vee B \in \mathcal{B}(X)$. Moreover, let $S$ be a closed convex subset of $X$. Then $S$ separates the sets $A$ and $B$ if and only if

$$
A+B \subset A \vee B \dot{+} S .
$$

Proof. Necessity. Let $a \in A$ and $b \in B$. Then there exist $\alpha, \beta \geq 0, \alpha+\beta=1$ such that $\alpha a+\beta b \in S$. Therefore, $a+b=\beta a+\alpha b+\alpha a+\beta b \in A \vee B \dot{+} S$. Hence $A+B \subset A \vee B \dot{+} S$.

Sufficiency. Fix any $a \in A$ and $b \in B$. Then it follows from the assumption $A+B \subset$ $A \vee B \dot{+} S$ that $a+B \subset A \vee B \dot{+} S$ and $b+A \subset A \vee B \dot{+} S$. Hence $B \subset A \vee B \dot{+} S-a$ and $A \subset A \vee B \dot{+} S-b$. By these inclusions and Lemma 2.4 we have $A \vee B \subset A \vee B \dot{+}$ $(S-a) \vee(S-b)=A \vee B \dot{+}(S+[-a,-b])$. Now we obtain from the order cancellation law (olc) that $0 \in S+[-a,-b]$. Hence $[a, b] \cap S \neq \emptyset$.

Corollary 3.2. Let $A, B \in \mathcal{B}(X)$ and $S$ be a closed convex subset of $X$. Then $S$ separates the sets $A$ and $B$ if and only if

$$
A+B \subset A \stackrel{\circ}{\vee} B \dot{+} S .
$$


Remarks. 1. The assumption $A \vee B \in \mathcal{B}(X)$ in Theorem 3.1 is essential. Note that in general the convex hull of the union of two bounded sets $A$ and $B$ is not a bounded set. Take for example

i) $\quad X=L^{p}[0,1]=\{f \mid f:[0,1] \rightarrow \mathbb{R}$, Lebesgue-measurable

$$
\text { such that } \left.\|f\|_{p}=\int_{0}^{1}|f(t)|^{p} d t<\infty, 0<p<1\right\} \text {. }
$$

Let $\mathbb{B}(0,1)=\left\{f \in L^{p} \mid\|f\|_{p} \leq 1\right\}$ be the unit ball. In [6] p. 161-162 it is shown that $\mathbb{B}(0,1)$ is a bounded set in $L^{p}[0,1]$ and that conv $\mathbb{B}(0,1)=L^{p}$. Now take $A=\{0\}, B=\mathbb{B}(0,1)$ then $A \vee B=L^{p}[0,1]$ is an unbounded set in $L^{p}[0,1]$ for $0<p<1$.

$$
X=l^{p}=\left\{x=\left.\left(x_{n}\right)_{n \in \mathbb{N}}\left|\|x\|_{p}=\sum_{i=1}^{\infty}\right| x_{i}\right|^{p}<\infty\right\} \quad \text { for } 0<p<1 .
$$

As in the space $L^{p}[0,1]$ the unit ball $\mathbb{B}(0,1)=\left\{x \in l^{p} \mid\|f\|_{p} \leq 1\right\}$ is bounded (see [6]; p. 161-162). Now denote for $i \in \mathbb{N}$ by $e_{i}=\left(\delta_{i j}\right)_{j \in \mathbb{N}}$ the i-th unit vector of $l^{p}$, where $\delta_{i j}$ is the Kronecker symbol. Obviously $e_{i} \in \mathbb{B}(0,1)$ for every $i \in \mathbb{N}$ but the elements

$$
x_{n}=\frac{1}{n}\left(e_{1}+\ldots+e_{n}\right) \in \operatorname{conv} \mathbb{B}(0,1)
$$

are unbounded since $\left\|x_{n}\right\|_{p}=n(1 / n)^{p}=n^{1-p}$. As in the case of $L^{p}$ we can take $A=$ $\{0\}, B=\mathbb{B}(0,1)$ and $A \vee B$ is an unbounded set in $l^{p}$ for $0<p<1$.

Note that it is obvious that in a locally convex vector space, the convex hull of bounded sets is also a bounded set.

2. Note that the separation law does not imply the separation of sets if one of the sets $A$ or $B$ is unbounded.

Take for instance $X=\mathbb{R}^{2}, A=\{(x, y) \mid 1 / x \leq y, x>0\}, B=\{(x, 0) \mid x \in[0,1]\}$ and $S=\{(0, y) \mid y \in[0,1]\}$. Then $A+B=A, A \vee B \dot{+} S=\{(x, y) \mid x, y \geq 0\}$. Hence $A+B \subset A \vee B+S$ but $S$ does not separate $A$ and $B$ (see Fig. 2).

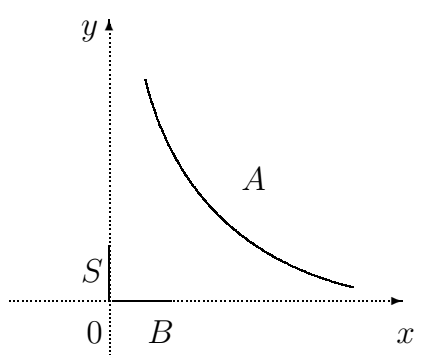

Fig. 2

THEOREM 3.3. In a locally convex vector space $(X, \tau)$ the order cancellation law and the separation law are equivalent.

Proof. Necessity. The proof of this part is identical to the proof of the second part of Theorem 3.1. 
Sufficiency. Now assume that the separation law (sl) is satisfied. Let $A, B, C \in \mathcal{A}(X)$ and assume that $B$ is bounded and $C$ is closed and convex. Now take any $a \in A$ and $b \in B$. From $A+B \subset B+C$ it follows that $a+(B-b) \subset(B-b) \dot{+} C$. Therefore, $\{0\}+(B-b) \subset(B-b) \vee\{0\} \dot{+}(C-a)$. Now from (sl) it follows that the set $C-a$ separates the sets $\{0\}$ and $B-b$. Hence $0=\alpha 0+\beta 0 \in C-a$ for some $\alpha, \beta \geq 0, \alpha+\beta=1$. So, we have $0 \in C-a$. Hence $A \subset C$.

By the same proof we get

TheOREM 3.4. Let $X$ be a topological vector space. Then for $\mathcal{B}(X)$ the order cancellation law and the separation law are equivalent.

4. Pairs of closed bounded convex sets. We will now consider inclusions of minimal representatives of the Rådström-Hörmander lattice of equivalence classes of pairs of nonempty closed bounded convex sets in a locally convex topological vector space $X$ (cf. [8], [9], [10]).

Therefore we assume that $(X, \tau)$ is a real locally convex vector space. An equivalence relation between pairs $(A, B),(C, D) \in \mathcal{B}^{2}(X)$ of closed bounded convex sets is given by the relation $(A, B) \sim(C, D)$ if and only if $A \dot{+} D=B \dot{+} C$ and the ordering in $\mathcal{B}(X)$ is extended to pairs by $(A, B) \leq(C, D)$ with $A \subseteq C, B \subseteq D$. From the order cancellation law it follows that " $\sim$ " is an equivalence relation in $\mathcal{B}^{2}(X)$. The equivalence class of $(A, B) \in \mathcal{B}^{2}(X)$ is denoted by $[A, B]$.

For compact convex sets we have the following result:

TheOREM 4.1. Let $(X, \tau)$ be a topological vector space. Then for any pair $(A, B) \in \mathcal{K}^{2}(X)$ there exists a pair $(C, D) \in[A, B]$ which is minimal.

Proof. Using the Kuratowski-Zorn lemma it is sufficient to show that for any totally ordered subset $\Sigma=\{(C, D) \in[A, B] \mid(C, D) \leq(A, B)\}$ of $[A, B]$ there exists an element $\left(A^{*}, B^{*}\right) \in[A, B]$ such that for any $(C, D) \in \Sigma$ the relation $\left(A^{*}, B^{*}\right) \leq(C, D)$ holds.

For any $\sigma=(C, D) \in \Sigma$ we will denote by $A_{\sigma}$ the set $C$ and by $B_{\sigma}$ the set $D$. The ordering on $\Sigma$ yields that $\sigma_{1} \leq \sigma_{2}$ if and only if $A_{\sigma_{1}} \subset A_{\sigma_{2}}$ and $B_{\sigma_{1}} \subset B_{\sigma_{2}}$.

Now we fix $\sigma_{0} \in \Sigma$ and define the sets $A^{*}=\bigcap_{\sigma \in \Sigma_{0}} A_{\sigma}$ and $B^{*}=\bigcap_{\sigma \in \Sigma_{0}} B_{\sigma}$, where $\Sigma_{0}=\left\{\sigma \in \Sigma \mid \sigma \leq \sigma_{0}\right\}$. By the Cantor intersection theorem (see [2]; Theorem 3.1.23) the set $A^{*}$ is nonempty. Moreover $A^{*}$ is a closed subset of $A_{\sigma_{0}}$ and hence it is compact. The convexity of $A^{*}$ follows immediately from the convexity of $A_{\sigma}$ for $\sigma \in \Sigma_{0}$. Since the same arguments hold for $B^{*}$ it follows that $\left(A^{*}, B^{*}\right) \in \mathcal{K}^{2}(X)$.

It remains to show that $\left(A^{*}, B^{*}\right) \in[A, B]$. By definition of the equivalence relation, for any pair $(C, D) \in[A, B]$ and for any $\sigma \in \Sigma_{0}$ the equation $A_{\sigma}+D=B_{\sigma}+C$ holds. This implies that $A^{*}+D \subseteq B_{\sigma}+C$ for every $\sigma \in \Sigma_{0}$. Hence for any $z \in A^{*}+D$ and any $\sigma \in \Sigma_{0}$ we can find a representation of the form $z=b_{\sigma}+c_{\sigma}$, where $b_{\sigma} \in B_{\sigma}$ and $c_{\sigma} \in C$. Since the net $\left\{b_{\sigma} \mid \sigma \in \Sigma_{0}\right\}$ is contained in the compact set $B_{\sigma_{0}}$ there exists a subnet $\left\{b_{\sigma_{\delta}} \mid \delta \in \Delta\right\}$ converging to some $b_{0} \in B_{\sigma_{0}}$. Hence for any neighborhood $U\left(b_{0}\right)$ of $b_{0} \in B_{\sigma_{0}}$ there exists an index $\delta_{0} \in \Delta$ such that for any $\sigma_{\delta} \leq \sigma_{\delta_{0}}$ we have $b_{\sigma_{\delta}} \in U\left(b_{0}\right)$ and therefore $B_{\sigma_{\delta}} \cap U\left(b_{0}\right) \neq \emptyset$. Now let $\sigma \in \Sigma_{0}$ be an arbitrary element. Since the set $\Sigma_{0}$ is totally ordered we have $\sigma_{\delta_{0}} \leq \sigma$ or $\sigma \leq \sigma_{\delta_{0}}$. In the first case $\sigma_{\delta_{0}} \leq \sigma$ we have 
$B_{\sigma_{\delta_{0}}} \subseteq B_{\sigma}$ and hence $B_{\sigma} \cap U\left(b_{0}\right) \neq \emptyset$. In the other case where $\sigma \leq \sigma_{\delta_{0}}$ we can find an index $\delta_{1} \in \Delta$ such that $\sigma_{\delta_{1}} \leq \sigma$ and for any $\sigma_{\delta} \leq \sigma_{\delta_{1}}$ we have $B_{\sigma_{\delta}} \cap U\left(b_{0}\right) \neq \emptyset$ and hence $B_{\sigma} \cap U\left(b_{0}\right) \neq \emptyset$. Thus we have shown that for any neighborhood $U\left(b_{0}\right)$ and any $\sigma \in \Sigma_{0}$ the set $B_{\sigma} \cap U\left(b_{0}\right)$ is not empty. Since the sets $B_{\sigma}$ are compact, it follows that $b_{0} \in B_{\sigma}$ for any $\sigma \in \Sigma_{0}$ and consequently $b_{0} \in B^{*}$. The subnet $\left\{c_{\sigma_{\delta}} \mid \delta \in \Delta\right\}$ converges to the point $z-b_{0}$ which by the compactness of $C$ belongs to $C$. Thus $A^{*}+D \subseteq B^{*}+C$ and by a similar argument we get $B^{*}+C \subseteq A^{*}+D$. Hence it follows that $\left(A^{*}, B^{*}\right) \in[A, B]$. The Kuratowski-Zorn lemma yields now that $[A, B]$ has a minimal element.

This is not longer true for closed bounded convex sets. Here we have:

TheOREM 4.2. Let $(X, \tau)$ be a reflexive locally convex vector space. Then every class $[A, B] \in \mathcal{B}^{2}(X) / \sim$ contains a minimal element $(C, D) \in[A, B]$.

Proof. In the case of finite-dimensional vector spaces, bounded closed sets are compact, and the theorem follows from Theorem 4.1. Let us denote by $\tau^{*}=\sigma\left(X, X^{*}\right)$ the weak topology for $X$. To avoid confusion, we will indicate during this proof the topology under consideration by an index at $\mathcal{B}$ and $\mathcal{K}$. In a reflexive locally convex vector space every bounded closed convex set $A \in \mathcal{B}_{\tau}(X)$ is compact in the topology $\tau^{*}$ and consequently belongs to $\mathcal{K}_{\tau^{*}}(X)$. Observe that every $A \in \mathcal{K}_{\tau^{*}}(X)$ is also closed in $\tau$ since $\tau^{*} \subset \tau$. Take any $(A, B) \in \mathcal{B}_{\tau}^{2}(X) \subset \mathcal{K}_{\tau^{*}}^{2}(X)$. Then

$$
A+B \in \mathcal{K}_{\tau^{*}}(X) \text { and } A \dot{+} B \in \mathcal{K}_{\tau}(X) .
$$

Therefore, the convex set $A+B$ is closed in $\tau$ and contained in $A \dot{+} B$, which is a bounded set in $X$ with respect to $\tau$. This implies that $A+B \in \mathcal{B}_{\tau}(X)$ and consequently $A+B=A+B$ holds in all reflexive topological vector spaces $(X, \tau)$. Hence $[A, B] \subset$ $[A, B]_{\tau^{*}} \in \mathcal{K}_{\tau^{*}}^{2}(X) / \sim$, where $[A, B]_{\tau^{*}}$ is the class of equivalent pairs of compact convex sets in the space $\left(X, \tau^{*}\right)$ which contains $(A, B)$. According to Theorem 4.1 , the equivalence class $[A, B]_{\tau^{*}}$ contains a minimal element $(C, D) \in \mathcal{K}_{\tau^{*}}^{2}(X)$ such that $C \subset A$ and $D \subset B$. Since $C, D$ are closed in $\tau$, convex and contained in bounded sets it follows that $(C, D) \in$ $\mathcal{B}_{\tau}^{2}(X)$. Moreover, $(C, D) \in[A, B] \subset[A, B]_{\tau^{*}}$. Therefore, $(C, D)$ is a minimal element in $[A, B]$ and, of course, $(C, D) \leq(A, B)$.

EXAMPLE 4.3. Let $l_{\infty}$ be the Banach space of all bounded real sequences endowed with the supremum norm $\left\|\left(x_{n}\right)\right\|=\sup _{n}\left|x_{n}\right|$ and let $c$ and $c_{0}$ be the subspaces of $l_{\infty}$ consisting of all convergent sequences resp. all sequences convergent to zero. Obviously $c_{0} \subset c \subset l_{\infty}$. Note that all three spaces are Banach spaces and that none of them is reflexive.

Let $\mathbb{B}(0,1)$ be the unit ball in $c_{0}$ and $A=\left\{a \in \mathbb{B}(0,1) \mid a_{n} \geq 0\right.$, for all $\left.n \in \mathbb{N}\right\}$. Put $B=-A$ and $A_{m}=\left\{a \in A \mid a_{1}=\ldots=a_{m}=\frac{1}{2}\right\}$ and $B_{m}=-A_{m}$ for $m \in \mathbb{N}$. Then $\left(A_{m}, B_{m}\right) \in \mathcal{B}^{2}\left(c_{0}\right)$ and $A+B_{m}=A_{m}+B$ for all $m \in \mathbb{N}$ and $A+B=\mathbb{B}(0,1)$. Thus $\left(A_{m}, B_{m}\right)$ is a decreasing chain of pairs in $[A, B]$, i.e.

$$
(A, B) \geq\left(A_{1}, B_{1}\right) \geq \ldots \geq\left(A_{m}, B_{m}\right) \geq \ldots
$$

with empty intersection, i.e. $\bigcap_{m} A_{m}=\bigcap_{m} B_{m}=\emptyset$. Now observe that the proof of Theorem 4.1 on the existence of minimal pairs of compact convex sets is based on the Cantor intersection property for compact sets. Therefore, we have: 
THEOREM 4.4. For each of the spaces $X=c_{0}, c$, and $l^{\infty}$ there exists a class $[A, B] \in$ $\mathcal{B}^{2}(X) / \sim$ which contains no minimal element.

OPEn question. The following question remains open: Given any non-reflexive topological vector space $X$. Does there exist an equivalence class $[A, B] \in \mathcal{B}^{2}(X) / \sim$ which contains no minimal elements?

For nonempty compact convex sets the situation is much simpler:

Let $(X, \tau)$ be a locally convex vector space. For $A \in \mathcal{K}(X)$ we consider a set $\mathcal{S} \subseteq$ $X^{*} \backslash\{0\}$ such that

$$
\overline{\operatorname{conv}\left(\bigcup_{f \in \mathcal{S}} H_{f}(A)\right)}=A .
$$

The sets $\mathcal{S} \subset X^{*} \backslash\{0\}$ of this type can be ordered by inclusion. A minimal element will be called a shape of $A$ and will be denoted by $\mathcal{S}(A)$. For a shape $\mathcal{S}(A)$ we consider subsets

$$
\mathcal{S}_{p}(A):=\left\{f \in \mathcal{S}(A) \mid \operatorname{card}\left(H_{f}(A)\right)=1\right\}
$$

which may be empty and

$$
\mathcal{S}_{l}(A):=\mathcal{S}(A) \backslash \mathcal{S}_{p}(A)
$$

Let $A, K \subset X$ be nonempty compact convex sets of a real locally convex topological vector space. Then $A$ is said to be a summand of $K$ if there exists a nonempty compact convex set $B \subset X$ with

$$
A+B=K
$$

for the Minkowski sum.

The criteria presented here are of two different types. The first type of criteria uses conditions which ensure that two compact convex sets are in a certain "general position", while the second type of criteria uses information about exposed points of the Minkowski sum of compact convex sets.

We begin with a criterion for minimality which is of the first type:

TheOREM 4.5. Let $X$ be a locally convex vector space, and let $A, B \subset X$ be nonempty compact convex sets. Let us assume that there is a shape $\mathcal{S}(A)$ of $A$ which satisfies the following conditions:

i) for every $f \in \mathcal{S}(A)$, card $\left(H_{f}(B)\right)=1$,

ii) for every $f \in \mathcal{S}_{l}(A)$ and every $b \in B$, the condition $\mathcal{S}_{l}(A)+\left(b-H_{f}(B)\right) \subseteq A$ implies $b=H_{f}(B)$,

iii) for every $f \in \mathcal{S}_{p}(A), H_{f}(A)-H_{f}(B) \in \mathcal{E}(A-B)$

or conversely, by interchanging $A$ and $B$. Then the pair $(A, B) \in \mathcal{K}^{2}(X)$ is minimal.

Proof. Let us assume that $A^{\prime} \subseteq A$ and $B^{\prime} \subseteq B$ are nonempty compact convex sets such that

$$
A+B^{\prime}=B+A^{\prime}
$$

Choose an element $f \in \mathcal{S}(A)$. Since

$$
H_{f}(A)+H_{f}\left(B^{\prime}\right)=H_{f}(B)+H_{f}\left(A^{\prime}\right)
$$

and since $H_{f}(B)=\{b\}$, this can be written as

$$
H_{f}(A)+H_{f}\left(B^{\prime}\right)=b+H_{f}\left(A^{\prime}\right) .
$$


Now choose an element $b^{\prime} \in H_{f}\left(B^{\prime}\right)$ and determine, for every extreme point $e \in \mathcal{E}\left(H_{f}(A)\right)$, an element $a_{e} \in H_{f}\left(A^{\prime}\right)$ such that

$$
e+b^{\prime}=b+a_{e}
$$

Now the following two cases are possible:

p) Let us assume that $f \in \mathcal{S}_{p}(A)$. Then $e-b=a_{e}-b^{\prime}$. Since, by condition iii), $e-b \in \mathcal{E}(A-B)$, we have $a_{e}=e$ and $b^{\prime}=b$. Hence $H_{f}\left(B^{\prime}\right)=H_{f}(B)=b$ and therefore, $H_{f}\left(A^{\prime}\right)=H_{f}(A)$.

l) Now we assume that $f \in \mathcal{S}_{l}(A)$. In this case we have for an arbitrary $b^{\prime} \in H_{f}\left(B^{\prime}\right)$ that

$$
H_{f}(A)+b^{\prime} \subseteq b+H_{f}\left(A^{\prime}\right)
$$

Therefore,

$$
H_{f}(A)+\left(b^{\prime}-b\right) \subseteq A^{\prime} \subset A
$$

and condition ii) gives $b=b^{\prime}$. Hence $H_{f}\left(A^{\prime}\right)=H_{f}(A)$.

Thus for all $f \in \mathcal{S}(A)$ we have $H_{f}\left(A^{\prime}\right)=H_{f}(A)$ and therefore,

$$
A^{\prime} \supseteq \operatorname{clconv}\left(\bigcup_{f \in \mathcal{S}} H_{f}\left(A^{\prime}\right)\right)=\operatorname{cl} \operatorname{conv}\left(\bigcup_{f \in \mathcal{S}} H_{f}(A)\right)=A,
$$

i.e. $A^{\prime}=A$. Now from the equality $A+B^{\prime}=B+A^{\prime}$ we get by the cancellation law that $B^{\prime}=B$, which completes the proof.

The next criterion for minimality is based on a sufficient condition on the indecomposability of a nonempty compact convex set and is formulated in terms of its exposed points. It uses a modified version of the Krein-Milman Theorem.

TheOREM 4.6. Let $X$ be a Banach space and let $(A, B) \in \mathcal{K}^{2}(X)$. If for every exposed point $a+b \in \mathcal{E}_{0}(A+B)$ with $a \in \mathcal{E}_{0}(A), b \in \mathcal{E}_{0}(B)$ there exists $b_{1} \in \mathcal{E}_{0}(B)$ or $a_{1} \in \mathcal{E}_{0}(A)$ such that $a+b_{1} \in \mathcal{E}_{0}(A+B)$ and $a-b_{1} \in \mathcal{E}(A-B)$ or $a_{1}+b \in \mathcal{E}_{0}(A+B)$ and $a_{1}-b \in \mathcal{E}(A-B)$, then $(A, B)$ is minimal.

Proof. Let $(A, B) \in \mathcal{K}^{2}(X)$. By Proposition 1.1 for every $f \in X^{*}$,

$$
H_{f}(A+B)=H_{f}(A)+H_{f}(B) .
$$

This implies the unique representation of every exposed point of $A+B$ as the sum of exposed points of $A$ and $B$.

Let us show that the pair $(A, B) \in \mathcal{K}^{2}(X)$ is minimal. Therefore, we choose a pair $\left(A^{\prime}, B^{\prime}\right) \in \mathcal{K}^{2}(X)$ with $A^{\prime} \subseteq A, B^{\prime} \subseteq B$ and $A+B^{\prime}=B+A^{\prime}$. For $a+b \in \mathcal{E}_{0}(A+B)$ we can assume without loss of generality that for $a \in \mathcal{E}_{0}(A)$ there exists $b_{0} \in \mathcal{E}(B)$ such that $a+b_{0} \in \mathcal{E}_{0}(A+B)$ and $a-b_{0} \in \mathcal{E}(A-B)$. Hence there exists a continuous linear functional $f_{0} \in X^{*}$ such that

$$
H_{f_{0}}(A+B)=\left\{a+b_{0}\right\} .
$$

By Proposition 1.1 we have $H_{f_{0}}(A)=\{a\}$ and $H_{f_{0}}(B)=\left\{b_{0}\right\}$. From $A+B^{\prime}=B+A^{\prime}=Y$ it follows that $H_{f_{0}}(A)+H_{f_{0}}\left(B^{\prime}\right)=H_{f_{0}}(B)+H_{f_{0}}\left(A^{\prime}\right)$. Hence there exist elements $a^{\prime} \in$ $H_{f_{0}}\left(A^{\prime}\right) \subseteq A$ and $b^{\prime} \in H_{f_{0}}\left(B^{\prime}\right) \subseteq B$ such that $a+b^{\prime}=b_{0}+a^{\prime}$. Since $a-b_{0} \in \mathcal{E}(A-B)$ it follows that $a=a^{\prime}, b_{0}=b^{\prime}$. From the equality $a=a^{\prime}$ it follows that $B+a \subseteq B+A^{\prime}=Y$. 
Hence $a+b \in Y$, and since $a+b \in \mathcal{E}_{0}(A+B)$ it follows from V. Klee's modification of the Krein-Milman Theorem (see [5]) that $A+B=Y$.

Hence by the cancellation law we have

$$
A+B=A^{\prime}+B, \quad \text { i.e. } \quad A=A^{\prime}
$$

and

$$
A+B=A+B^{\prime}, \quad \text { i.e. } B=B^{\prime} .
$$

Therefore, $(A, B) \in \mathcal{K}^{2}(X)$ is minimal.

ExAmple 4.7. To illustrate these criteria, we will give two typical examples for $X=\mathbb{R}^{2}$.

i) Let $R$ be a positive real number and put $x=\frac{1}{2} \sqrt{3} R, \quad y=\frac{1}{2} R, a_{1}=(0, R), a_{2}=$ $(x,-y), \quad a_{3}=(-x,-y)$ and let $A=a_{1} \vee a_{2} \vee a_{3}$ and $B=-A$. It follows from Theorem 4.5 that the pair $(A, B)$ i.e. the $S t a r$ of David (see Fig. 3 ) is minimal.

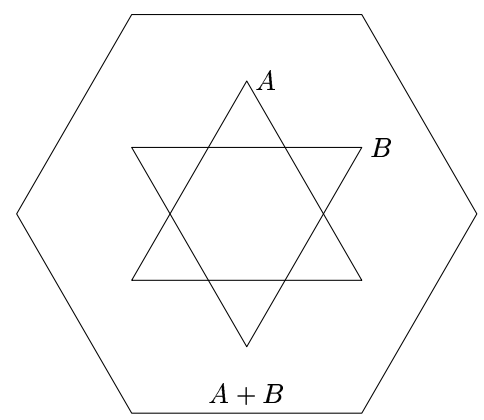

Fig. 3

ii) Let $R>0$ be given and let us define the linear map

$$
T: \mathbb{R}^{2} \rightarrow \mathbb{R}^{2} \quad \text { by } \quad T\left(x_{1}, x_{2}\right)=\left(-x_{2}, x_{1}\right) .
$$

For $x_{0}=\left(\frac{1}{2} \sqrt{2} R, 0\right)$ take the balls $K_{1}=\mathbb{B}\left(x_{0}, R\right), K_{2}=\mathbb{B}\left(-x_{0}, R\right)$. Put $A=K_{1} \cap$ $K_{2}, \quad B=T(A)$. Then $A+B=A-B=B((0,0), R)$. It is easy to see that the conditions stated in Theorem 4.6 give the minimality of the pair $(A, B)$ of orthogonal lenses (see Fig. 4).

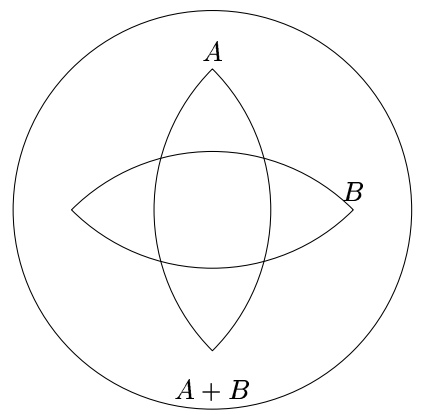

Fig. 4 
It was proved by S. Scholtes and J. Grzybowski that minimal pairs in the twodimensional space are unique up to translation. This is not true for higher dimensions as shown by a counter-example of J. Grzybowski (cf. [3], [15]).

In December $2000 \mathrm{~S}$. Rolewicz posed the question whether the set of equivalent minimal pairs, which are not related by translation may be finite and of cardinality greater than one.

Recently J. Grzybowski and R. Urbański gave a negative answer to this question.

TheOREM 4.8. Let $(X, \tau)$ be a topological vector space and $\left(A_{1}, B_{1}\right),\left(A_{2}, B_{2}\right) \in \mathcal{K}^{2}(X)$ be two equivalent minimal pairs which are not related by translation. Then there exists an uncountable family $\left(A_{\lambda}, B_{\lambda}\right), \lambda \in \Lambda$ of minimal pairs that are all equivalent to $\left(A_{1}, B_{1}\right)$ and no $\left(A_{\lambda}, B_{\lambda}\right)$ is a translate of $\left(A_{\mu}, B_{\mu}\right)$ for $\lambda \neq \mu$.

5. The invariance of dimension. In this section we discuss invariants which belong only to minimal pairs of compact convex sets and do not belong to the whole class. Such invariants are the affine dimension and codimension of the union of a minimal pair of compact convex sets. We begin with the following result:

TheOREM 5.1. Let $(X, \tau)$ be a topological vector space and let $(A, B),(C, D) \in \mathcal{K}^{2}(X)$ be equivalent pairs. Furthermore let us assume that the pair $(C, D) \in \mathcal{K}^{2}(X)$ is minimal. If $A \cup B \subset X_{0} \subset X$, where $X_{0}$ is a closed subspace of $X$, then there exists a point $x_{0} \in X$ such that $C \cup D \subset X_{0}+x_{0}$.

Proof. By assumption the pairs $(A, B),(C, D) \in \mathcal{K}^{2}(X)$ are equivalent, i.e.

$$
A+D=B+C \text {. }
$$

Hence for every $a_{0} \in A$ and $d_{0} \in D$ there exist points $b_{0} \in B$ and $c_{0} \in C$ with $a_{0}+d_{0}=$ $b_{0}+c_{0}$. Therefore, we have

$$
\left(A-a_{0}\right)+\left(D-d_{0}\right)=\left(B-b_{0}\right)+\left(C-c_{0}\right) .
$$

If we put $A_{0}=A-a_{0}, B_{0}=B-b_{0}, C_{0}=C-c_{0}$ and $D_{0}=D-d_{0}$, then we can rewrite equation (5.1) as

$$
A_{0}+D_{0}=B_{0}+C_{0} \text { with } 0 \in A_{0} \cap B_{0} \cap C_{0} \cap D_{0}
$$

Now we consider the sets $C^{\prime}=C_{0} \cap X_{0}$ and $D^{\prime}=D_{0} \cap X_{0}$. By equation (5.2), for every $x \in A_{0}$ and $y \in D^{\prime}$ there exist elements $b \in B$ and $c \in C$ such that

$$
x+y=\left(b-b_{0}\right)+\left(c-c_{0}\right) .
$$

Since

$$
c-c_{0}=(x+y)-\left(b-b_{0}\right) \in X_{0}+X_{0}+X_{0}+X_{0}=X_{0},
$$

we obtain $c-c_{0} \in C_{0} \cap X_{0}=C^{\prime}$, and hence $A_{0}+D^{\prime} \subset B_{0}+C^{\prime}$. Analogously $B_{0}+C^{\prime} \subset$ $A_{0}+D^{\prime}$ and hence

$$
\left(A_{0}, B_{0}\right) \sim\left(C_{0}, D_{0}\right) \sim\left(C^{\prime}, D^{\prime}\right) .
$$

Since the pair $\left(C_{0}, D_{0}\right)$ is minimal, we get

$$
C-c_{0}=C^{\prime} \text { and } D-d_{0}=D^{\prime} .
$$


Hence, we have $C-c_{0} \subset X_{0}$ and $D-d_{0} \subset X_{0}$. These inclusions imply that

$$
C \subset X_{0}+c_{0}=\left(X_{0}-b_{0}\right)+b_{0}+c_{0}=X_{0}+\left(b_{0}+c_{0}\right)
$$

and

$$
D \subset X_{0}+d_{0}=\left(X_{0}-a_{0}\right)+a_{0}+d_{0}=X_{0}+\left(a_{0}+d_{0}\right) .
$$

Hence $C \subset X_{0}+x_{0}, D \subset X_{0}+x_{0}$ with $x_{0}=\left(b_{0}+c_{0}\right)$.

REMARK. Let $(X, \tau)$ be a locally convex vector space and $C \in \mathcal{K}(X)$ be a nonempty compact convex subset. Then for every $y \in C$ the set

$$
C_{y}=\overline{\operatorname{span}(C-y)}=\operatorname{cl}\left(\left\{z \in X \mid z=\sum_{i=1}^{n} \lambda_{i}\left(c_{i}-y\right), \quad c_{i} \in C, n \in \mathbb{N}, \lambda_{i} \in \mathbb{R}\right\}\right)
$$

is the smallest closed linear subspace containing $C-y$ or equivalently the intersection of all closed linear subspaces containing $C-y$. The affine hull of $C$ is given by

$$
\operatorname{aff} \operatorname{hull}(C)=C_{y}+y
$$

and it is independent of the choice of $y \in C$. The affine dimension and codimension are defined by

$$
\operatorname{dim} \operatorname{aff}(C)=\operatorname{dim}\left(C_{y}\right), \quad \operatorname{codim} \operatorname{aff}(C)=\operatorname{codim}\left(C_{y}\right)=\operatorname{dim}\left(X / C_{y}\right) .
$$

In particular:

Corollary 5.2. Let $(X, \tau)$ be a topological vector space and $(A, B),(C, D) \in \mathcal{K}^{2}(X)$ be equivalent minimal pairs. Then

$$
\operatorname{dim} \operatorname{aff}(A \cup B)=\operatorname{dim} \operatorname{aff}(C \cup D), \quad \operatorname{codim} \operatorname{aff}(A \cup B)=\operatorname{codim} \operatorname{aff}(C \cup D) .
$$

Open question. Apart from the affine dimension and codimension of the union of a minimal pair of compact convex sets, no further invariants for minimal pairs of compact convex sets are known.

\section{References}

[1] V. F. Demyanov and A. M. Rubinov, Quasidifferential Calculus, Optimization Software Inc., Publications Division, New York, 1986.

[2] R. Engelking, General Topology, PWN-Polish Scientific Publishers, Warszawa, 1977.

[3] J. Grzybowski, Minimal pairs of compact convex sets, Archiv der Mathematik 63 (1994), $173-181$.

[4] L. Hörmander, Sur la fonction d'appui des ensembles convexes dans un espace localement convex, Arkiv för Matematik 3 (1954), 181-186.

[5] V. Klee, Extremal structure of convex sets II, Math. Zeitschrift 69 (1958), 90-104.

[6] G. Köthe, Topologische Lineare Räume, Grundlehren der Mathematischen Wissenschaften 107, Springer Verlag, Berlin, 1966.

[7] D. Pallaschke, P. Recht and R. Urbański, On locally Lipschitz quasidifferentiable functions in Banach spaces, Optimization 17 (1986), 287-295.

[8] D. Pallaschke, S. Scholtes and R. Urbański, On minimal pairs of compact convex sets, Bull. Polish Acad. Sci. Math. 39 (1991), 1-5. 
[9] D. Pallaschke and R. Urbański, Some criteria for the minimality of pairs of compact convex sets, Zeitschrift für Operations Research 37 (1993), 129-150.

[10] D. Pallaschke and R. Urbański, Reduction of quasidifferentials and minimal representations, Mathem. Programming Series A 66 (1994), 161-180.

[11] D. Pallaschke and R. Urbański, Pairs of Compact Convex Sets-Fractional Arithmetic with Convex Sets, Math. Appl. 548, Kluwer, Dordrecht, 2002.

[12] A. G. Pinsker, The space of convex sets of a locally convex space, Trudy Leningrad Engineering-Economic Institute 63 (1966), 13-17.

[13] H. Rådström, An embedding theorem for spaces of convex sets, Proc. Amer. Math. Soc. 3 (1952), 165-169.

[14] S. Rolewicz, Metric Linear Spaces, PWN and Reidel, Warszawa-Dordrecht, 1984.

[15] S. Scholtes, Minimal pairs of convex bodies in two dimensions, Mathematika 39 (1992), 267-273.

[16] R. Urbański, A generalization of the Minkowski-Rådström-Hörmander Theorem, Bull. Acad. Polon. Sci. Sér. Sci. Math. Astr. Phys. 24 (1976), 709-715. 